\title{
THEORY OF LATERAL BANDGAP VARIATION ACHIEVABLE BY STRAIN ENGINEERING IN PATTERNED SUBSTRATE STRAIN EPITAXY
}

\author{
Jasprit Singh \\ Center for High-Frequency Microelectronics \\ Department of Electrical Engineering and Computer Science \\ The University of Michigan, Ann Arbor, MI 48109-2122 USA
}

(Received $30 \mathrm{July}, 1990$ )

\begin{abstract}
Energy minimization considerations are used to estimate the strain tensor for pseudomorphic structures grown on a patterned substrate. We show that if a material $B$ is deposited below critical thickness in a hole of width $W$ in a substrate A the strain is biaxial unless $W \leq 50 \AA$, when the strain becomes hydrostatic. For $\mathrm{W}>50 \AA$ if the material $\mathrm{A}$ is deposited on top of the layer $\mathrm{B}$, as the thickness of $\mathrm{A}$ is increased, the lattice matched overlayer $A$ gradually readjusts the strain causing the strain in region $B$ to change from biaxial to hydrostatic. For reasonable film thickness we find that hydrostatic strain can be produced for patterns with widths up to $0.3 \mu \mathrm{m}$. Since the bandgap changes considerably when the strain changes from biaxial to hydrostatic, this concept can be used to produce lateral variation in the bandgap of heterostructures in single step epitaxy.
\end{abstract}

Strained epitaxy has added another exciting parameter to the band tailoring attainable in heterostructures. The strain produced in the usual strained epitaxy is such that the in-plane lattice constant of the overlayer fits the substrate while the perpendicular lattice constant responds via the Poisson effect leading to biaxial strain. As shown in Fig. 1, it is difficult to obtain lateral variation of bandgap during single step epitaxy in lattice matched or strained epitaxy. Of course ex-situ methods can be devised using lithographic techniques combined with regrowth to produce lateral variations. These techniques are usually quite intricate and difficult to control. Lateral variation would be particularly advantageous in the area of optical devices where it would then be possible to design sources, modulators and detectors responding to different wavelengths on the same chip.

We examine the possibility of laterally modifying the strain tensor via strained epitaxy on patterned substrates. If the strain tensor in the overlayer changes from one region of the substrate to another in a controllable manner, the deformation potential theory can then be used to estimate the range of lateral bandgap tailoring possible. We will examine the conditions under which the strain tensor can be modified from the usual biaxial kind produced in flat substrate epitaxy.
For this we examine the growth of material $B$ in a hole of dimensions $\mathrm{L}$ and $\mathrm{W}$ on a substrate of material $\mathrm{A}$ as shown in Fig. 2(a).

The misfit between the overlayer and the substrate is

$$
\epsilon=\frac{a_{A}-a_{B}}{a_{A}} \quad \text { where }
$$

Let $a_{B}=$ lattice constant of the free overlayer. $a_{A}=$ lattice constant of the substrate.

For focus we assume $a_{A}<a_{B}$

The average force constants of the layers are $\mathrm{C}_{11}$, $\mathrm{C}_{12}, \mathrm{C}_{44}$. We choose these constants to have a value of $12.0 \times 10^{11}$ dynes $/ \mathrm{cm}^{2}, 5.5 \times 10^{11}$ dynes $/ \mathrm{cm}^{2}$ and $5 \times$ $10^{11}$ dynes $/ \mathrm{cm}^{2}$ respectively. These represent typical values for semiconductors.

We will focus only on the material $B$ in the hole and not on the remaining region of the substrate. We will assume that the thickness $d_{1}$ of the material $B$ is smaller than the critical thickness so that no dislocations are generated at the overlayer substrate interface. Let us consider the energy of the system after deposition of material $B$ and examine whether the strain is biaxial or hydrostratic. 

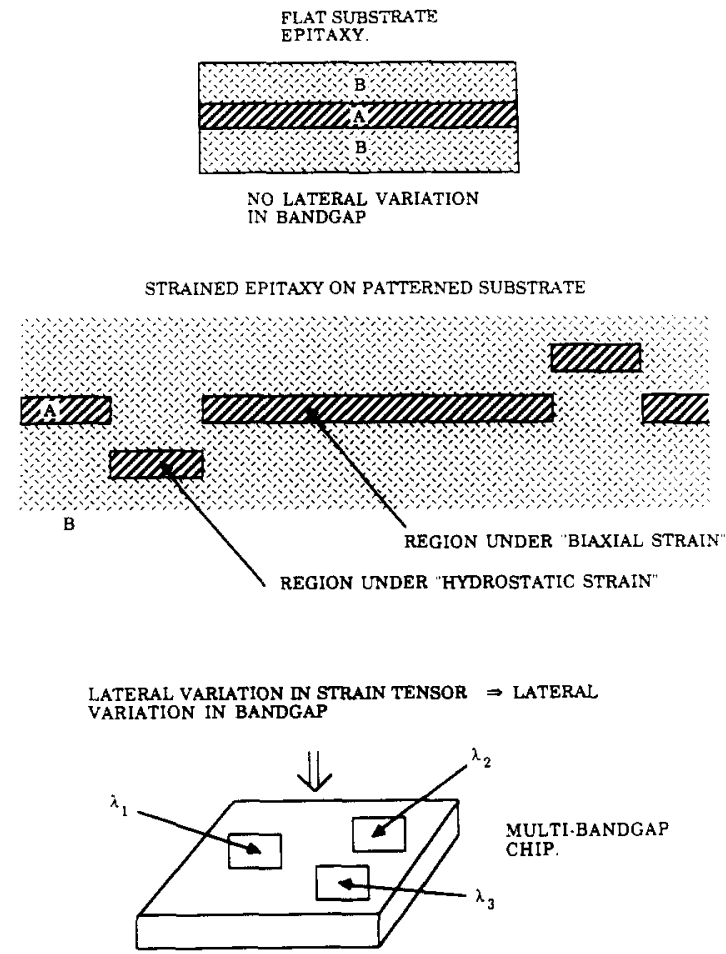

\section{Figure 1}

A schematic showing how in flat substrate epitaxy, it is difficult to obtain abrupt changes in lateral bandgap, while in strained epitaxy in patterned substrates, it may be possible to change the strain tensor from biaxial to hydrostatic. This could lead to devices on the same chip with varying optical wavelength response.

The general strain energy is given by

$$
\begin{aligned}
E= & \frac{1}{2} C_{11}\left(\epsilon_{x x}^{2}+\epsilon_{v y}^{2}+\epsilon_{x y}^{2}\right)+C_{12}\left(\epsilon_{x x} \epsilon_{y y}+\right. \\
& \left.\epsilon_{y y} \epsilon_{z x}+\epsilon_{x x} \epsilon_{z z}\right)+\frac{1}{2} C_{44}\left(\epsilon_{x y}^{2}+\epsilon_{y z}^{2}+\epsilon_{x z}^{2}\right)
\end{aligned}
$$

where " $\mathrm{a}$ " is the average lattice constant.

We compare two cases as shown in Fig. 2(b). For the biaxial strain case the strain in the growth plane $(\mathrm{x}-\mathrm{y})$ is:

$$
\begin{aligned}
& \epsilon_{x x}=\epsilon=\epsilon_{y \nu} \\
& \epsilon_{z z}=-\frac{2 C_{12}}{C_{11}} \epsilon
\end{aligned}
$$

Where $\epsilon$ is the misfit between A and B. For purely biaxial strain the strain energy is

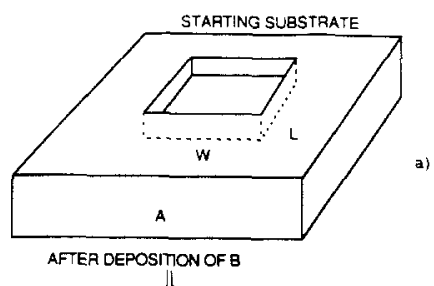

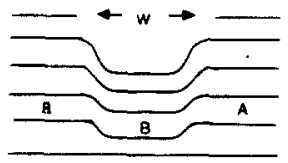

BIAXIAL STRAIN EDGE STRAIN
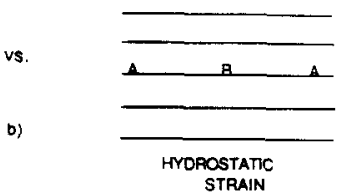

STRAIN

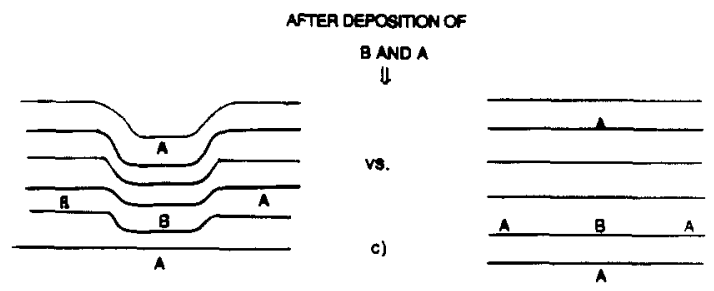

Figure 2

A schematic for the various cases considered in the text: a) the patterned substrate with a hole of length $L$ and width $W ; b$ ) Two extreme strain conditions showing biaxial strain and hydrostatic strain after B has been deposited; c) After deposion of lattice matched overlayer $A$, the structure which was under biaxial strain (after deposition of B) could remain so, or could "pop out" to produce hydrostatic strain in the region $B$. We assume that the lattice constant of $B$ is smaller than that of $\mathbf{A}$.

$$
E=\epsilon^{2}\left(C_{11}+C_{12}-\frac{2 C_{12}^{2}}{C_{11}}\right) L W d_{1}
$$

However, clearly if the strain is biaxial, we will set dislocations at the edges of the "hole". Thus at the edges the strain will be close to hydrostatic to maintain the proper bonding. This will cause an excess strain given by the hydrostatic strain tensor $\left(\epsilon_{x x}=\epsilon_{y y}=\epsilon_{x z}\right.$ $=\epsilon ; \epsilon_{x y}=\epsilon_{y x}=\epsilon_{x x}=0$ ),

$$
E \cong\left(\frac{3}{2} C_{11}+3 C_{12}\right) \epsilon^{2}\left(2 L_{1} d_{1} a+2 W d_{1} a\right) .
$$

The total energy for the case $a$ is then (after using the values of the force constants)

$$
E \sim 12.5 \epsilon^{2} L W d_{1}+69 \epsilon^{2}\left(L d_{1}+W d_{1}\right) a\left(10^{11} \text { ergs }\right) .
$$


In contrast, for the case $b$, where the strain is hydrostatic the strain energy is

$$
E \sim 34.5 \epsilon^{2} L W d_{1} \quad\left(10^{11} \text { ergs }\right)
$$

It is quite clear that the case $a$ has a lower energy unless $W$ is of the order of a few lattice constants. For wider lines, the strain continues to be biaxial, except in the regions very close to the edges of the patterned hole. This places very severe contraints on changing the strain tensior from biaxial to hydrostatic.

Let us now consider the case where a thickness $d_{2}$ of material $A$ is deposited on top of the material $B$ and $W$ is $>10 a$. We show below that for small values of $\mathrm{d}_{2}$, it is favorable that the overlayer $A$ remain curved at a curvature determined by the biaxial strain in film $B$ (left hand schematic of Fig. 2(c)). However, as the thickness $d_{2}$ continues to increase, it is eventually favorable for the entire film to "pop up" and become flat leading to a hydrostatic strain in the region $B$ (right hand schematic of Fig. 2(c)). To establish the conditions for this to happen, we again examine the energy of the film near the patterned hole. The shear energy of the film $\mathbf{A}$ for the case where the film is curved as in the left hand schematic of Fig. 2(c) is;

$$
E_{\text {shear }} \cong\left(20 \frac{\epsilon^{2} d_{1}^{2}}{W^{2}}\right) L W d_{2} \quad\left(10^{11} \text { ergs }\right)
$$

for our choice of $\mathrm{C}_{11}, \mathrm{C}_{12}$, and $\mathrm{C}_{44}$. The shear is assumed to be

$$
\sim 2.0 \epsilon \frac{d_{1}}{W}
$$

For the film to "pop up" as shown in the right hand schematic of Fig. 2(c), the shear energy has to be larger than the excess energy needed to produce hydrostatic strain. This condition yields the result,

$$
20 \epsilon^{2} d_{1}^{2} d_{2} \frac{L}{W}>34.5 \epsilon^{2} \times L W d_{1}
$$

or roughly,

$$
W^{2} \leq 0.6 d_{1} d_{2}
$$

Thus the growth of the lattice matched overlayer allows one to increase the width of the "hole" to values larger than 10a and obtain hydrostatic strain. For realistic overlayer thickness of $<5 \mu \mathrm{m}$, and $d_{1} \leq 200 \AA$, we find that

$$
W \leq 0.3 \mu m
$$

This condition is, of course, much more accessible than the earlier condition of $40-50 \AA$. In fact, submi- cron technology currently is capable of such linewidths. It must be noted that in the above discussions we are assuming that the system is able to reach its energy minimum during growth. Of course, this will depend upon growth conditions since it is well known that epitaxial techniques can produce metastable states. However, we also know that for proper growth conditions (i.e. slow growth rates) the critical thickness theory based upon equilibrium conditions is quite applicable for dislocation generation where large kinetic barriers have to be overcome. Unlike dislocation generation during growth where mass movement within the crystal is required during growth, for the cases discussed above there is no such requirement on rearrangement of chemical bonds. This allows us to expect the above theory to be valid under normal epitaxy. The change of the strain tensor from biaxial on a flat region to hydrostatic in the patterned region (with $W \leq 0.3 \mu \mathrm{m}$ ) can produce a large change in the bandgap of the material $B$. Notice that $\mathrm{W} \sim 0.3 \mu \mathrm{m}$ will not produce any bandgap change due to quantization effects. Those effects become important only if $W \leq 200 \AA$.

An estimate of the bandgap changes can be easily made. ${ }^{2-4}$

The HH bandgap change is:

$\Delta E_{g a p}^{H H}=-\left\{a\left(\epsilon_{x x}+\epsilon_{y y}+\epsilon_{x x}\right)+\frac{b}{2}\left(\epsilon_{x x}+\epsilon_{y y}-2 \epsilon_{x x}\right)\right\}(11)$

The LH bandgap change is:

$\Delta E_{g a p}^{L H}=-\left\{a\left(\epsilon_{x x}+\epsilon_{y y}+\epsilon_{x x}\right)-\frac{b}{2}\left(\epsilon_{x x}+\epsilon_{y y}-2 \epsilon_{z x}\right)\right\}(12)$

Where $a$ and $b$ are deformation potentials considering the two extremes of biaxial and hydrostatic strain we have

$$
\text { Biaxial : } \begin{aligned}
\Delta E_{\text {gap }}^{H H} & =-\left\{2 a \epsilon_{11}\left(\frac{C_{11}-C_{12}}{C_{11}}\right)\right. \\
& \left.+b \epsilon_{11}\left(\frac{C_{11}+2 C_{12}}{C_{11}}\right)\right\} \\
\Delta E_{\text {gap }}^{L H} & =-\left\{2 a \epsilon 11\left(\frac{C_{11}+C_{12}}{C_{11}}\right)\right. \\
& \left.-b \epsilon_{11}\left(\frac{C_{11}+2 C_{12}}{C_{11}}\right)\right\}
\end{aligned}
$$

which are the usual conditions for strain induced bandgap effects for flat substrate epitaxy. The other extreme gives,

$$
\text { Hydrostatic : } \begin{aligned}
\Delta E_{g a p}^{H H} & =-3 a \epsilon_{11} \\
\Delta E_{g a p}^{L H} & =-3 a \epsilon_{11}
\end{aligned}
$$

To get a quantitative idea of the amount of lateral bandgap change possible through the lateral strain tai- 
loring, we evaluate the changes for $\mathrm{In}_{0.2} \mathrm{Ga}_{0.8} \mathrm{As}$, a composition which has a $1.4 \%$ lattice mismatch with GaAs. The parameters used are $a=8.4 \mathrm{ev}$ and $b=-1.7 \mathrm{ev} .^{3}$

The effective bandgap changes by $\sim 250 \mathrm{meV}$ as the strain changes from biaxial to hydrostatic. In addition, if the strained layer is incorporated in a quantum well, the quantization effect must be accounted for. This is not expected to vary much across the substrate since the total quantization effect is about an order of magnitude smaller than the strain induced bandgap changes.

The change in bandgap calculated above is very large and could be of great value if exploited in optoelectronic devices. It must be remembered, however, that it is calculated under the simplifying assumptions detailed in the paper. At present, little is known about the details of strained epitaxy in patterned substrates in terms of how the strain is accommodated. Our calculations indicate clearly that this area of strained epitaxy should be explored by experimentalists since it may provide a versatile technique for novel optoelectronic device design. However, careful experiments must be carried out to study the strain tensor and its lateral variation in patterned strained epitaxy.
Acknowlegement - This work is supported by the Office of Naval Research and a grant from the IBM Corp.

\section{References:}

1. See for example, Introduction to Solid State Physics, C. Kittel (John Wiley and Sons, New York, Fourth Edition). These discussions are not present in later editions of this book.

2. G. L. Bir and G. E. Pikus, Symmetry and Strain Induced Effects in Semiconductors, John Wiley and Sons, New York (1974).

3. H. Kato, N. Iguchi, S. Chika, M. Nakayama and N. Sano, J. Appl. Phys. 59, 588 (1986).

4. S. C. Hong, G. P. Kothiyal, N. Debbar, P. Bhattacharya, and J. Singh, Phys. Rev. B 37, 878 (1988). 